https://helda.helsinki.fi

\title{
The Prodromal Microbiome
}

\section{Scheperjans, Filip}

2018-01

Scheperjans , F 2018 , ' The Prodromal Microbiome ' , Movement Disorders, vol. 33 , no. 1 , pp. 5-7 . https://doi.org/10.1002/mds.27197, https://doi.org/10.1002/mds.27197, https://doi.org/10.1002/mds.27197

http://hdl.handle.net/10138/235503

https://doi.org/10.1002/mds.27197

unspecified

publishedVersion

Downloaded from Helda, University of Helsinki institutional repository.

This is an electronic reprint of the original article.

This reprint may differ from the original in pagination and typographic detail.

Please cite the original version. 


\title{
The Prodromal Microbiome
}

\author{
Filip Scheperjans, MD, PhD (D)* \\ Department of Neurology, Helsinki University Hospital and Department of Clinical Neurosciences (Neurology), \\ University of Helsinki, Helsinki, Finland
}

In human microbiome research, reproducibility is crucial because the microbial ecosystem is heavily influenced by environmental factors and highly variable between individuals. This poses challenges when designing such studies and recruiting suitable subjects. Furthermore, the employed methodology of data acquisition and analysis can be highly customized, and differences in the work flow may introduce biases and hamper comparisons between studies.

In the last 2 years, the gut microbiome has gained increasing attention in the Parkinson's disease research community because of its possible implication in the prominent gastrointestinal disturbances frequently present from the earliest stages of PD and the ascension hypothesis of PD-related neuropathology. ${ }^{1}$ So far, 9 original publications have reported gut microbiota alterations in PD. ${ }^{2-10}$ As could be expected, the subject selection criteria, laboratory protocols, and bioinformatical and statistical pipelines differed considerably between studies. Sample size ranged from 38 to 327 subjects. Most studies used $16 \mathrm{~S}$ ribosomal RNA amplicon sequencing to assess the community structure of the gut microbiota, whereas 2 studies relied on qPCR analysis of preselected bacterial taxa. These approaches are relatively cost effective and allow a description of differences in the relative abundances of bacterial taxa between study groups (ie, the community structure).

Despite the above-mentioned limitations and considerable differences in their methodology and results, it is encouraging that the abovementioned studies showed clear overlaps with respect to the bacterial taxa differentially abundant between PD patients and

${ }^{*}$ Correspondence to: Filip Scheperjans, Department of Neurology, Helsinki University Central Hospital, Haartmaninkatu 4, 00290 Helsinki, Finland; filip.scheperjans@hus.fi

Relevant conflicts of interest/financial disclosures: F.S. is listed as inventor on patent application WO2015/181449A1 and is founder and CEO of Neurolnnovation Oy.

Funding agencies: none.

Received: 11 September 2017; Accepted: 13 September 2017

Published online 30 October 2017 in Wiley Online Library (wileyonlinelibrary.com) DOI: 10.1002/mds.27197 control subjects. Increased abundance of Akkermansia $^{2,3,5-7}$ and Lactobacillus ${ }^{2,4,6,8,9}$ and decreased abundance of Prevotella $a^{2,4,5,7,8}$ in PD subjects were reported in 5 studies. Other reproducible findings were reduced abundance of Faecalibacterium ${ }^{5,6,8,10}$ (4 studies) and Blautia $^{3,6,10}$ (3 studies) and increased abundance of Bifdobacterium $^{5,6,8}$ (3 studies) in PD.

So by now, that the gut microbial community structures of PD patients and controls differ seems to be reasonably well established, with several bacterial genera showing consistent abundance alterations in multiple studies. However, it was not known whether these alterations precede the development of PD or whether they are rather a consequence of a dysfunctional gut associated with PD.

In this issue of Movement Disorders, HeintzBuschart et al add an important piece of evidence to the field by demonstrating that indeed gut microbiome alterations may precede the motor symptoms of PD. ${ }^{11}$ In addition to patients with established PD, they studied a group of subjects suffering from a well-defined prodromal syndrome of $\mathrm{PD}$, namely, rapid eye movement sleep behavior disorder (RBD). Because RBD is associated with an up to $90 \%$ risk of developing a neurodegenerative disorder later on (mostly synucleinopathies), it is considered a promising criterion for selection of patients for premotor trials of diseasemodifying treatments. ${ }^{12}$

In their study, Heintz-Buschart et al employed stateof-the-art analytical pipelines to assess microbiome community structure in nasal and fecal samples and aimed to account for potential confounders such as constipation and medications. ${ }^{11}$ The rationale for the analysis of nasal microbiota was that, like the gastrointestinal tract, the nasal cavity has been suggested to constitute a port of entry for a possible pathogenic agent. ${ }^{1}$ For the first time, in this study, nasal microbial communities were directly compared with fecal communities. As has been previously described, the nasal bacterial community was sparse and showed high interindividual variation. ${ }^{11,13}$ Operational taxonomic units (OTUs) common to the nasal and gastrointestinal microbiota were found, likely attributable to nasal discharge being swallowed. However, none of these OTUs differed significantly between study groups. 
Regarding taxa that were differentially distributed between PD patients and controls, effects of sex and medications seemed to explain more of the observed differences than PD itself. Therefore, the authors agree with a previous study that the biomarker potential of nasal microbiota for PD seems limited. ${ }^{11,13}$

The analysis of fecal microbiota at the genus level showed an increased abundance of Akkermansia in the feces of PD patients, in line with several previous studies. ${ }^{2,3,5-7,11}$ Also, Clostridium XlVb and Anaerotruncus were enriched in PD subjects, but showing also increased abundance in RBD subjects. However, in contrast to Akkermansia, these were not confirmed by the more stringent Analysis of composition of microbiomes (ANCOM) analysis. Eight further genera showed altered abundance in RBD. Of these, only decreased abundance of Prevotella was confirmed by ANCOM. On the OTU level, OTUs assigned to Akkermansia (increased in PD), Prevotella, and Clostridiales (both decreased in PD), and Flavonifractor (increased in PD and RBD) were confirmed by ANCOM. In addition to the Flavonifractor OTU, 9 other OTUs showed significant differences in abundance in the same direction in $\mathrm{PD}$ and $\mathrm{RBD}$ subjects versus controls, but were not confirmed by ANCOM: OTUs assigned to Prevotellaceae, Olsenella, Bacteroides, Ruminococcaceae, Sutterella, and an $\alpha-$ proteobacterium were decreased in PD and RBD, whereas OTUs assigned to Anaerotruncus and Clostridium XlVa were increased in PD and RBD.

So the most robust findings from this complex analysis seem to be an increased abundance of Akkermansia in PD and a decreased abundance of Prevotella in PD (1 OTU) and RBD (genus), together with decreased abundance of a Clostridiales OTU in PD and an increased abundance of a Flavonifractor OTU in PD and RBD. So not only do these results support previous findings regarding altered abundance of Akkermansia and Prevotella in PD, but for the first time it has been shown that regarding Prevotella such alterations may already be detected in the prodromal period. ${ }^{2-8}$ The relevance of the Clostridiales and Flavonifractor OTUs, which showed comparably low mean abundance compared with the previous ones remains to be established.

The pure comparison between patient groups, although the cornerstone of the analysis, can be influenced by other, possibly unknown confounding factors that are differentially distributed between the study groups. Associations between bacterial abundance and clinical symptoms of the PD spectrum can add plausibility to these findings and can support, although not prove, causal connections. Of the most robustly altered genera in PD, Akkermansia was negatively associated with nonmotor symptom burden, as measured by the MDS UPDRS I. ${ }^{11}$ Several other genera and OTUs including Anaerotruncus and Clostridium $X I V b$ showed associations with motor and nonmotor symptoms. ${ }^{11}$ This supports previous reports of connections between motor and nonmotor symptoms and gut microbiota. $^{2,14}$

Virtually all RBD patients will go on to develop a synucleinopathy. ${ }^{12}$ Thus, although not formally a longitudinal study, this important article gives some insight into the likely time course of microbiome changes in PD. It suggests that a decreased abundance of Prevotella precedes the motor phase, at least in PD patients suffering from premotor RBD. Because RBD patients were drug naive in relation to PD medications, this study strongly suggests that reduced Prevotella abundance is not explained by PD medications, a question that was difficult to assess in previous case-control studies of medicated patients. Furthermore, these changes do not seem to be a consequence of the development of overt motor deficits and corresponding dopaminergic denervation.

The article by Heintz-Buschart et al is an important step toward a better understanding of the role of microbiota in PD. That microbiome alterations could already be detected in the premotor period in a subgroup of PD patients makes gut microbiota appear as an even more promising PD biomarker than before. Now it seems possible that future therapeutic trials aimed at microbiome modulation may already be applied in premotor patients.

However, one major question still remains unanswered, and that is the specificity of these exciting findings. As in all previous studies on microbiota and PD, constipation is an important potential confounder. Although most studies, including the one by HeintzBuschart et al, tried to assess constipation using questionnaires and accounted for it in the analysis, this problem has so far not been sufficiently addressed. The main problem is that in PD objective colonic dysfunction seems to be far more prevalent than patientreported constipation symptoms. ${ }^{15}$ No specific constipation questionnaire has been formally validated in PD patients using objective measurements of gut function as a gold standard. As in this study, a significant proportion of RBD patients also reported constipation, the same limitation also applies to the RBD-related findings. Gut microbiome composition, in particular abundance of Akkermansia and Prevotella, clearly correlates with gastrointestinal symptoms, transit time and stool consistency. ${ }^{14,16}$ Therefore, it is not clear whether these changes are merely a consequence of PD-related gut dysfunction or whether they are a contributing factor. However, because constipation itself is associated with an increased risk of PD, this knowledge gap does not reduce the importance of these findings for a better understanding of PD pathogenesis.

Based on the attributed functions of the bacterial taxa now reproducibly associated with PD, gut 
mucosal integrity, short-chain fatty acids, and inflammation could be important pathways linking microbiota and PD. However, depending on environmental and intrinsic factors, bacterial taxa can contribute in different ways to the ecosystem of the gut. Therefore, differences in the taxonomic composition of microbiota do not necessarily reflect functional differences. ${ }^{17}$ Predictions of the functional potential can be calculated based on microbial abundance and data on microbial genomic content from public databases. However, these predictions may be inaccurate as they, for example, do not account for microbes without sequenced genomes. ${ }^{11}$

As Heintz-Buschart et al concluded, only metagenomic analyses based on shotgun sequencing can truly inform the functional capacity of the microbiota. ${ }^{11}$ Furthermore, metagenomics allows the extension of the scope of analysis to other microbes, but for these purposes it has so far only been used in 1 PD study. ${ }^{7}$ Multiomic approaches studying the activity of the microbiome and its interactions with the genome and immune system of the host will hopefully help a better understanding and treatment PD in the future.

Now that we have been introduced to the prodromal microbiome, it is time to figure out what it is doing to our patients.

\section{References}

1. Hawkes CH, Del Tredici K, Braak H. Parkinson's disease: a dualhit hypothesis. Neuropathol Appl Neurobiol 2007;33(6):599-614.

2. Scheperjans F, Aho V, Pereira PA, et al. Gut microbiota are related to Parkinson's disease and clinical phenotype. Mov Disord 2015; 30(3):350-358

3. Keshavarzian A, Green SJ, Engen PA, et al. Colonic bacterial composition in Parkinson's disease. Mov Disord 2015;30(10):13511360.
4. Hasegawa S, Goto S, Tsuji H, et al. Intestinal dysbiosis and lowered serum lipopolysaccharide-binding protein in Parkinson's disease. PLoS One 2015;10(11):e0142164.

5. Unger MM, Spiegel J, Dillmann KU, et al. Short chain fatty acids and gut microbiota differ between patients with Parkinson's disease and age-matched controls. Parkinsonism Relat Disord 2016;32:6672 .

6. Hill-Burns EM, Debelius JW, Morton JT, et al. Parkinson's disease and Parkinson's disease medications have distinct signatures of the gut microbiome. Mov Disord 2017;32(5):739-749.

7. Bedarf JR, Hildebrand F, Coelho LP, et al. Functional implications of microbial and viral gut metagenome changes in early stage LDOPA-naive Parkinson's disease patients. Genome Med 2017;9(1): 39-017-0428-y.

8. Petrov VA, Saltykova IV, Zhukova IA, et al. Analysis of gut microbiota in patients with Parkinson's disease. Bull Exp Biol Med 2017;162(6):734-737.

9. Hopfner F, Kunstner A, Muller SH, et al. Gut microbiota in Parkinson disease in a northern german cohort. Brain Res 2017;1667: 41-45.

10. Li W, Wu X, Hu X, et al. Structural changes of gut microbiota in Parkinson's disease and its correlation with clinical features. Sci China Life Sci 2017 [Epub ahead of print].

11. Heintz-Buschart A, Pandey U, Wicke T, et al. The nasal and gut microbiome in Parkinson's disease and idiopathic rapid eye movement sleep behavior disorder. Mov Disord 2018;33:88-98.

12. Howell MJ, Schenck CH. Rapid eye movement sleep behavior disorder and neurodegenerative disease. JAMA Neurol 2015;72(6): 707-712.

13. Pereira PAB, Aho VTE, Paulin L, et al. Oral and nasal microbiota in Parkinson's disease. Parkinsonism Relat Disord 2017;38:61-67.

14. Mertsalmi TH, Aho VTE, Pereira PAB, et al. More than constipation - bowel symptoms in Parkinson's disease and their connection to gut microbiota. Eur J Neurol 2017 [Epub ahead of print].

15. Knudsen K, Fedorova TD, Bekker AC, et al. Objective colonic dysfunction is far more prevalent than subjective constipation in Parkinson's disease: A colon transit and volume study. J Parkinsons Dis $2017 ; 7(2): 359-367$.

16. Vandeputte D, Falony G, Vieira-Silva S, et al. Stool consistency is strongly associated with gut microbiota richness and composition, enterotypes and bacterial growth rates. Gut 2016;65(1):57-62.

17. Cho I, Blaser MJ. The human microbiome: at the interface of health and disease. Nat Rev Genet 2012;13(4):260-270. 